\title{
PERSPECTIVE FONCTIONNELle DE LA PHRASE ET LINGUISTIQUE DU TEXTE FRANÇAISE. L'ÉCOLE de PRAGUE... PASSANT PAR LA LORRAINE
}

\author{
Guy ACHARD-BAYLE \\ Université de Lorraine \& CREM-Praxitexte
}

\begin{abstract}
En): In this paper, I want to present the personality of Robert Martin, whose works are not well known outside France, and analyse the important role he played in the early eighties for the revival of linguistics - considering at the same time the rising influence of his colleagues Bernard Combettes, Jean-Michel Adam and Michel Charolles, that is to say the influence they had on the introduction of text linguistics in France, specifically thanks to the creation in Metz of a new journal in the second part of the seventies: Pratiques. If Robert Martin first wanted to introduce natural logics in formal semantics, he was not insensible to the Prague School's legacy either; but for him, the manner to introduce a discursive dimension or level in his linguistic model, that is to say a textual one between sentence and utterance, finally was a way to affirm and preserve the independence of semantics from pragmatics - the growing part of pragmatics in linguistics or the so called "language sciences" at the beginning of the same decade.
\end{abstract}

Keywords (En): Natural logic; text linguistics; logical subject; topic; presupposition

\section{Introduction : cadrage épistémologique et mise en perspective géo-historique}

La présente étude fait suite à une conférence («Du Cercle linguistique de Prague à la grammaire de texte française ») prononcée à l'Université de Bohême du Sud (UBS) lors du colloque international Pour une Europe des sciences sans frontières, organisé à l'occasion du vingtième anniversaire de la Révolution de velours, en novembre 2009.

Ce texte publié en anglais par l'UBS (sur CD-Rom) a été repris, revu et complété, en français, pour la publication des Actes de la journée organisée à l'Université catholique de Milan (UCM) le 26 octobre 2010', par les trois partenaires institutionnels du réseau «Cercle de Prague, linguistique textuelle, sémantique » : l'UBS, l'UCM et l'Université Paul Verlaine Metz (UPVM) ${ }^{2}$.

Ces deux premières communications se proposaient de considérer l'apport en France du Cercle linguistique de Prague d'une part à la linguistique générale en France, d'autre part, et plus précisément, à une grammaire de texte ou une linguistique du texte «à la française », i.e. telle que cette dernière s'est particulièrement développée au tournant des années 1980 dans les universités lorraines, à Metz et Nancy, et peut-être davantage encore autour de la revue Pratiques créée peu avant (au milieu des années $70^{3}$ ) par des enseignants proches et des enseignants-chercheurs de ces universités ${ }^{4}$.

\footnotetext{
${ }^{1}$ Voir G. ACHARD-BAYle (2010).

${ }^{2}$ Devenue depuis le $1^{\text {er }}$ janvier «université de Lorraine» (UdL) avec les trois établissements supérieurs de Nancy: Universités Henri Poincaré, Nancy 2, Institut National Polytechnique de Lorraine (INPL).

${ }^{3}$ Voir en ligne http://www.pratiques-cresef.com/ quelques articles du numéro fondateur ( ${ }^{\circ} 1-2$ mars 1974) ; dans l'un d'eux, J.-F. Halté \& A. Petitjean transcrivent le cours qu'ils ont fait «à l'Université Nouvelle de Metz » («Pour une théorie de l'idéologie d'un manuel scolaire, le Lagarde et Michard :
} 
À l'UBS en 2009, comme à l'UCM en 2010, je me suis également attaché à mettre en perspective ces échanges «Est-Ouest », dans ce que j'appelais au-delà d'une histoire, une « géographie des idées linguistiques ».

Ici, mon propos sera différent : il sera davantage question de sémantique et de pragmatique que de linguistique générale, voire de grammaire de texte, au sens où celle-ci, dans la lignée de la grammaire générative des années 70 (cf. les travaux de T. van Dijk par exemple ${ }^{5}$ ), a partie liée avec les modèles syntaxiques structuralistes, fussent-ils, par force (la génération des textes), macrosyntaxiques, soit inter- ou transphrastiques.

Ainsi, j'envisagerai le cas très particulier que représentent, pour l'éclosion d'une linguistique du texte «à la française » dans le cadre géo-historique posé, les travaux de B. Combettes, notamment Pour une grammaire textuelle. L'exemple des progressions thématiques qu'il publie en 1983, et l'ouvrage que R. Martin fait paraître la même année, Pour une logique du sens, qui introduit dans le cadre d'une sémantique de tradition vériconditionnelle, ou plus largement en logique naturelle, les dimensions textuelle et discursive pour un calcul du sens véridictionnel (autre «mot valise » dont je m'expliquerai plus bas).

Ceci dit, B. Combettes ayant participé au colloque dont on publie ici les Actes, et, avant même, à la journée Conscila organisée à Paris en 2010 (voir note 4), tout comme J.-M. Adam qui accorde à son collègue et à son ouvrage une place de première importance, je ne m'attarderai pas à répéter - sans doute en moins bien ce qu'ils ont pu eux-mêmes dire puis écrire à ce propos.

Je préfère donc reporter mon attention sur l'auteur que $j$ 'ai introduit après B. Combettes, R. Martin; il y a un intérêt tout particulier à s'y attacher ici, un double intérêt : d'une part, son nom n'est pas très familier aux linguistes, disons, d'obédience pragoise ; d'autre part, si R. Martin a joué un rôle considérable pour les recherches linguistiques, en l'occurrence dans la création d'un centre de recherches linguistiques à l'université de Metz dans les années 70, donc peu après sa création (cf. note 3), son renom lui vient plus de ses travaux en sémantique logique qu'en linguistique textuelle.

Or, R. Martin, qui publie la même année que B. Combettes son ouvrage majeur, n'est pas davantage éloigné de lui au plan géographique; ce qui peut expliquer que dans son ouvrage de sémantique logique, ou de logique naturelle, il se fasse l'écho de l'avancée des travaux pragois en matière de perspective fonctionnelle; et qu'il le fasse en faveur de l'émergence d'une théorie linguistique qui rende compte du transphrastique, qui prenne en compte le texte, au-delà de la phrase.

le cas Diderot », Pratiques 1-2, 43-64) : les deux, revue et université messine, vont donc quasiment de pair.

${ }^{4}$ Voir les communications de J.-M. Adam et de B. Combettes à la journée Conscila de mai 2010 (à par. in Verbum, revue de l'université de Lorraine). Sur le site de la revue, cité note 3, on trouvera des fac-similés de quelques numéros et articles « historiques »: B. COMBETTES \& J. FressON (1975), M. Charolles (1976), J.-M. Adam (1977).

${ }^{5}$ Voir B. Combettes (1976). 
Dans cette perspective, de la phrase au texte ${ }^{6}$, et que l'on pourra comparer à la perspective pragoise, R. Martin distingue, avec précaution et finesse, phrase et énoncé, cotexte et contexte, sens vériconditionnel et sens véridictionnel.

Ce qui est déjà tout un programme ; et un apport majeur, sans aucun doute, à une sémantique logique naturelle telle que R. Martin la conçoit et l'instruit : à partir des modèles ou des schèmes phrastiques de la langue (voir encore les deux citations de notre conclusion), vers les énoncés actualisés, contextualisés, de cette langue, autrement dit tels qu'ils y apparaissent naturellement.

Mais R. Martin montre en outre, en forme de passage continu entre deux niveaux de représentation et d'analyse sémantiques : le textuel et le discursif ou le cotextuel et le contextuel, le rapport qui existe entre le thème et le présupposé de l'énoncé ; ainsi, au-delà de la phrase, la sémantique s'ouvre à la dimension du texte, et la sémantique textuelle, elle, s'articule à la pragmatique linguistique.

Ma contribution comprendra deux parties : après avoir rapidement rappelé le propos de mes communications de 2009 à l'UBS et 2010 à Milan, j'aborderai l'étude proprement dite des textes et des théories de R. Martin suivant l'angle d'analyse donné, à l'intersection de la sémantique logique de la proposition et de la linguistique du texte ; la conclusion proposera également, et paradoxalement, une ouverture sur une autre époque de l'École de Prague : la première si je suis le classement en trois époques que propose, ici et ailleurs, S. Raynaud ; autrement dit ma conclusion sera rétroactive, sinon « anachronique ».

\section{Genèse et parcours d'une «linguistique de texte », de Prague à Metz et Nancy}

Il est difficile sinon de faire la genèse du moins de retrouver la raison exacte d'une influence qui prend en l'occurrence la forme d'une renaissance ou d'une redécouverte. La question est néanmoins intéressante pour l'histoire des idées, l'épistémologie et la philosophie des sciences. Le cas qui va nous intéresser ici, et tel qu'il nous a intéressés à l'UBS pour le vingtième anniversaire de la Révolution de velours en 2009, est un bel exemple de circulation des idées sans frontières, suivant le titre du colloque qui nous accueillait.

En 1983, donc, deux ouvrages (COMBETTES 1983, MARTIN 1983) paraissent en français, qui vont bouleverser le paysage linguistique français, caractérisé d'un côté par le structuralisme chomskyen ou néo-chomskyen; de l'autre par le fonctionnalisme «néo-pragois » d'A. Martinet, dont on peut dire, du point de vue que je représente ici, qu'il est, comme le précédent modèle, attaché au système de la langue plus qu'aux réalisations en discours ${ }^{7}$.

Je relève une coïncidence de date. Mais les deux ouvrages ont une autre caractéristique commune, qui n'est pas la moindre : chacun des titres commence

\footnotetext{
${ }^{6}$ C'est aussi le sous-titre d'une série de manuels scolaires Bâtir une grammaire. Vers la maîtrise de la langue. De la phrase au texte, publiés par B. Combettes avec J. Fresson et R. Tomassone à partir de 1979 chez Delagrave à Paris.

${ }^{7}$ Discours est pris ici dans un sens générique qui rassemble les manifestations orales ou écrites des énonciations contextualisées et cotextualisées. Par contre, je n'entre pas dans le détail des différences textes-discours telles que les développe notamment J.-M. Adam (par exemple dans ses derniers travaux, voir bibliographie).
} 
par Pour, les deux auteurs proposant un renouveau ${ }^{8}$ qui participe de l'évolution, de la transformation qui s'opère alors de la linguistique aux/en sciences du langage : $\mathrm{R}$. Martin œuvre en faveur d'une logique du sens naturelle, en rupture avec la logique formelle, décontextualisée; quant à B. Combettes, en sa présence à l'UBS en 2011, j'ai hésité à parler de ce qu'il a voulu faire en 1983 : je renvoie donc par précaution à ce qu'il a dit lui-même, et éventuellement de lui-même, notamment dans sa conférence de mai 2010 pour Conscila .

La comparaison entre les deux ouvrages ne s'arrête pas à leur date et à leur titre. On peut en effet se demander encore ce qui réunit les deux ouvrages et leurs auteurs dans un même élan de renouveau en faveur de la langue naturelle et de productions linguistiques complexes et contextualisées : dans cette perspective, c'est l'appui qu'ils prennent, sans paradoxe néanmoins, on va le montrer, sur une unité linguistique issue de la tradition logique philosophique.

\section{La proposition, sujet logique et psychologique}

Tous deux partent de la proposition de la tradition philosophique ${ }^{10}$ : au sens logique, la proposition est considérée, comme «l'expression du jugement ». Si l'on suit en effet Descartes et les grammairiens français du XVII ${ }^{\text {ème }}$ siècle (notamment A. Arnauld \& C. Lancelot), mais aussi N. CHOMSKY et sa Linguistique cartésienne (1966), la proposition logique est fondée rationnellement sur un sujet (ce dont on prédique) et un attribut (ce qu'on prédique).

Cela «tourne rond», mais cela tourne aussi un peu « en rond»! Il faudra donc en dire plus... Pour aller dans ce sens, il faudra trouver une passerelle entre système et discours ou texte ${ }^{11}$, et de manière plus générale, comme on l'a dit, ménager le passage dans les années 80 de la linguistique aux sciences du langage. Cette passerelle sera fournie par les Pragois : aussi bien B. Combettes que R. Martin disent à quel point ils leur doivent, de V. Mathesius à J. Firbas, de J. Vachek à F. Daneš. ${ }^{12}$

Ainsi la notion de proposition logique, qui fonde la prédication comme «effigie de la raison » selon les Cartésiens, doit être révisée : il n'est plus dès lors question de sujet logique ou d'argument, ni de prédicat logique ou d'attribut; il est question, avec et après Prague, de sujet et de prédicat psychologiques; j'en déduis donc qu'il s'agit également d'interaction, d'intercompréhension, de partage $\mathrm{du}$ sens : le sujet (ce dont on affirme) devient un thème (ce dont on parle), le prédicat (ce qu'on affirme) un rhème (ce qu'on en dit); le «jugement » ou

\footnotetext{
${ }^{8}$ Je pense aussi à la rénovation pédagogique qu'a représentée le travail de B. Combettes : s'il eut une influence remarquable sur l'enseignement du français (voir note 6), ce fut aussi à l'étranger ; c'est ainsi que je l'invitais moi-même, dans ces mêmes années 80, en Autriche pour y conduire des séminaires de formation de professeurs de français; voir une dizaine d'années plus tard B. COMBETTES (1992).

${ }^{9}$ Voir supra note 3 .

${ }^{10}$ Depuis B. Combettes a publié une nouvelle étude sur le sujet (cf. COMBETTES, 2011).

${ }^{11}$ Discours-texte : je reviens plus bas sur leur distinction.

${ }^{12}$ Voir ces quatre auteurs «pragois» en bibliographie. On pourrait encore citer K. Bühler et N. Troubetzkoy, comme le fait B. ComBETTES au tout début de son ouvrage de 1983: les deux linguistes se connurent à l'université de Vienne ; nouvelle preuve qu'une histoire peut s'enrichir d'une géographie des idées.
} 
l'assertion de la tradition cartésienne ou logique cède ainsi le pas à la parole, au langage en action et en situation, aux discours et aux textes, tels qu'ils sont à prendre, dans leur contexte; la phrase entre alors dans une autre perspective: fonctionnelle; on pourra, dans l'environnement «lorrain», parler de perspective macrosyntaxique, transphrastique, ou simplement textuelle.

En guise de transition vers ma deuxième partie, je dirai encore que, si la logique classique a ainsi été soumise à l'épreuve du réel ou du quotidien linguistique, autrement dit à l'épreuve des modalités et du «flou », alors la valeur de vérité (fondamentale dans son modèle formel) a pu céder le pas aux fonctions du langage qui dépassent la seule nécessité du calcul de la vérité ; on s'écarte du calcul formel ou formaliste des conditions de vérité (la vériconditionnalité) pour s'intéresser aux manières de dire, de poser et de supposer la vérité : autrement dit on s'intéresse à la véridictionnalité, telle que l'a développée notamment M. CHAROLLES (1997).

Ma deuxième partie va maintenant être consacrée à R. Martin que, dans la perspective et le cercle qui nous réunit ici, je voudrais faire connaitre ou reconnaitre ${ }^{13}$.

\section{Univers de croyance, communauté présuppositionnelle : perspectives psycho/logiques du sens}

Je commence par reprendre l'essentiel de ce qui précède: pour R. MARTIN (1983), donc, l'enjeu est de fonder une autre sémantique, même si celle-ci puise à la logique au-delà de ses fondements cartésiens : B. Russell, L. Wittgenstein, A. Tarski, P. Strawson, W.V.O. Quine, tous ces auteurs sont dans la bibliographie de son ouvrage. Mais cette sémantique intègre également les avancées linguistiques des années 60-70, en matière notamment d'énonciation et d'argumentation, ou plus largement de pragmatique : voir, toujours dans la même bibliographie, J. Austin, J. Searle, O. Ducrot.

Le projet de R. Martin est donc d'intégrer à la sémantique de tradition logique, jusqu'en ces développements analytiques, ce qui paradoxalement manquait le plus à ces derniers si l'on considère le linguistic turn de la philosophie et la logique germanique et anglo-saxonne d'une grande première moitié du $20^{\text {ème }}$ siècle : voir par ex. le projet de Strawson de fonder une «métaphysique descriptive; ce qui manquait donc à ce linguistic turn, ce n'était pas tant le langage naturel que ses manifestations concrètes, dans des énoncés et des langues ; quant à R. MARTIN, s'il continue de plaider pour une sémantique du vrai, il y intègre dès le départ (1983 : 13) ce qu'il appelle «trois notions fondamentales » : la vérité floue, les mondes possibles et les univers de croyance, lesquels deviendront en quelque sorte sa «marque de fabrique ». L'ensemble, surtout si l'on conjugue la première et la dernière des trois notions, lui permet alors de rendre compte d'un énoncé comme :

Cette 2CV n'en est pas une.

${ }^{13}$ Voir côté français F. DuvAL (éd. 2011), côté tchèque T. HosKOVEC (2010) et O. PEŠEK (2010). 
C'est un énoncé logiquement étrange, voire absurde au nom des principes de vérité et de non-contradiction, et pourtant, en termes linguistiques ou plus exactement pragmatiques, si l'on prend en compte l'interprétation en contexte, cet énoncé est parfaitement compréhensible ou pertinent, même si en l'occurrence, c'est-à-dire tel qu'on le présente hors contexte, il faut, pour rendre cette pertinence, restituer toute une situation, et son interaction: celle où un(e) automobiliste s'adresserait à un(e) garagiste qui se retrouvent, à la fin de réparations, devant une ancienne «vraie » $2 \mathrm{CV}$, maintenant restaurée et donc faite de pièces de récupération, plus ou moins d'origine, et de ce fait plus ou moins reconnaissable.

Cet exemple est capital selon moi, parce qu'il illustre parfaitement ce que nous concevons depuis lors, dans la communauté linguistique, comme une «logique du sens »; mais il l'est encore plus pour moi, en ce qu'il annonce avec une dizaine d'années d'avance la problématique de la référence évolutive: si celle-ci puise également à une vieille tradition logique, philosophique, voire métaphysique (voir l'énigme du bateau de Thésée $e^{14}$ ), elle prendra son essor dans le cadre du projet Cognisciences L'anaphore et son traitement, dans le « Grand Est » de la France au début des années 90 , sous l'impulsion de M. Charolles et G. Kleiber. ${ }^{15}$

Ainsi, on peut sans aucun doute avancer que, sans le recours à la sémantique logique floue de R. Martin, on n'aurait pu concevoir la référence évolutive en termes linguistiques, pas plus qu'on n'aurait su observer et analyser la façon dont, linguistiquement, on (re)saisit les référents évoluant, se déformant, conceptuellement et temporellement ${ }^{16}$.

Pour revenir à l'exemple de la $2 \mathrm{CV}$, on voit l'importance qu'il y a, en vue de l'interprétation, d'introduire le contexte: le contexte au sens le plus large, puisqu'il concerne ici aussi l'interaction entre deux sujets et les objets qui peuplent leur (notre) monde avec mais aussi envers et contre eux (et nous); autrement dit, on voit l'importance d'ouvrir le domaine ou le champ sémantico-logique au pragmatique comme facteur de relativité et de relativisation ${ }^{17}$.

\footnotetext{
14 Je cite ici la quatrième de couverture de Stéphane FERRET qui lui consacre un ouvrage (1996) : «Un paradoxe et plus encore une énigme : l'identité à travers le temps. Que dire, par exemple, du bateau de Thésée, bateau perpétuellement réparé dont les sophistes d'Athènes se demandaient, au fur et à mesure que les pièces en étaient modifiées ou remplacées, s'il s'agissait encore du même bateau ? » J'ai longuement travaillé à la question : ACHARD-BAYLE (2001).

15 Voir la publication en 1994 à Metz du n ${ }^{\circ} 19$ des Recherches Linguistiques : L'anaphore associative: aspects linguistiques, psycholinguistiques et automatiques, C. SCHNEDECKER, M. Charolles, G. Kleiber, J. DAvid (éds). Voir également dans mon ouvrage cité (2001 : 113), les origines de ma recherche (1993) dans le cadre de ce projet.

16 Cette problématique a permis de produire un nombre assez considérable d'études, voire d'ouvrages, mais elle a subitement disparu du panorama des recherches linguistiques à l'orée des années 2000. Je pense que cette disparition est due au fait que nous nous occupions aussi de référence en termes philosophiques et logiques, autrement dit suivant une position « réaliste » qui a sans doute dérangé l'immanentisme qui domine en sciences du langage comme en linguistique dans la tradition saussurienne, si l'on en croit les vives critiques « constructivistes » que nous avons eu à affronter.

${ }^{17}$ Pour autant R. Martin ne répètera pas, dix ans après, la pragmatique dans la veine rhétoricoargumentative d'O. DUCROT (1972) ; j'y reviendrai dans G. ACHARD-BAYLE (2012).
} 
Tout cela va donc mener R. Martin à s'interroger (l'un des premiers ?) sur le passage d'un domaine ou d'une discipline à l'autre: de la sémantique à la pragmatique ; c'est le titre de son dernier chapitre (dans l'édition de 1983), j'y reviendrai, mais c'est aussi une préoccupation (et une évolution après son premier ouvrage de 1976) qu'il annonce dès l'introduction et dont il démontre dès lors les implications : le propos de R. Martin sera d'acheminer la théorie et l'analyse sémantiques de la proposition à l'énoncé ; en se souvenant (je rappelle ici la partie précédente) d'une part, que dans une tradition logique qui irait de la Grammaire Générale \& Raisonnée à N. Chomsky, la proposition logique est constituée d'un sujet (ce dont on prédique) et d'un attribut (ce qu'on prédique); d'autre part, comment ces notions logiques ont laissé la place à celles de sujet et de prédicat psychologiques.

La proposition et donc la logique classique ou formelle sont ainsi soumises à l'épreuve du réel ou du quotidien, autrement dit des sujets, des intentions et des modalités : on passe ainsi du calcul formel des conditions de vérité ou de la vériconditionnalité (soit: "À quelles conditions la proposition $\mathrm{P}$ peut-elle être vraie ? »), à la véridictionnalité qui concerne les manières de dire, de poser et de supposer le vrai - elle concerne aussi, dans ses développements plus récents, les portées de la vérité : voir le néo-fonctionnalisme de M. CHAROLLES (1997) et ses cadres discursifs.

Pour résumer, on pourrait donc dire que l'apport particulier de R. Martin est d'avoir donné une assise et une dimension psychologiques à la sémantique, et de les faire fructifier linguistiquement dans un modèle de tradition logique. Je vais terminer sur ce point, en m'attachant d'abord à la notion de contexte puis à celle de thème.

\section{De la sémantique du contexte à la logique du thème}

On a vu que R. MARTIN veut intégrer la pragmatique à la sémantique, mais on sent bien dès son introduction de 1983, qui ne change d'ailleurs pas sur ce point en 1992, qu'il se méfie de la première, revenant à diverses reprises à des bases sémantiques. Pour lui en effet, s'il faut bien « rendre compte de l'extrême diversité des faits » (op. cit. : 15) ou encore de la « diversité du langage naturel » (ibid.), il n'en reste pas moins que la pragmatique "peut être difficilement 'intégrée' » (ibid.). Les relations « dépendantes », « labiles », « contingentes » (ibid.) qu'elle instaure et les situations discursives dans lesquelles il faut les prendre en compte sont «aussi variables que les situations elles-mêmes », tandis que la sémantique est le domaine du «nécessaire », de l' " invariant », du « stable », du «prévisible » et du «calculable» (ibid.); à l'inverse, «le domaine de l'interprétation pragmatique » est « difficile à contrôler» (op. cit : $14^{18}$ ).

Ceci dit, il faut bien rendre compte des interprétations (ce que l'on a voulu dire), au-delà du sens (le dit) : les interprétations relèvent des énoncés, qui sont le lieu du vrai (et du faux), quand les phrases, autrement dit leur sens, ne sont que le lieu des conditions de vérité (op . cit. 1993 : 13-14, 1992 : 16-17). La partition est

\footnotetext{
${ }^{18}$ Je souligne contrôler avant d'y revenir en conclusion. Notons par ailleurs que l'ensemble est repris tel quel dans la seconde éd. de 1992 (op. cit. : 15-17).
} 
faite certes ${ }^{19}$, mais comment passer de la composante sémantique à la composante pragmatique (op . cit. $1993: 15,1992: 18)$ ? En distinguant dans la première deux «fonctions » (le terme, par sa connotation métalinguistique «jakobsonienne», n'est évidemment pas le fait d'un choix aléatoire ${ }^{20}$ ) : (i) la «fonction phrastique », « initiale » (ibid.), qui élabore le sens comme lieu des conditions de vérité (ou de l'acceptabilité sémantique); et (ii) la «fonction discursive», qui «assure l'insertion de la phrase dans le discours », « en créant ainsi la cohésion textuelle » (ibid.).

Il importe de relever alors, dans un tel système d'analyse et d'interprétation évolutives (voir les flèches dans le schéma qui suit), que c'est la seconde fonction qui «maintient la continuité thématique » (ibid.); et de remarquer encore que cette mention d'une «continuité thématique » est à cette date sans doute inédite, du moins en France, dans un ouvrage de sémantique logique, car il s'agit bien, toujours, de définir là le domaine sémantique :

\section{Ainsi se dégage un schéma à trois paliers :}

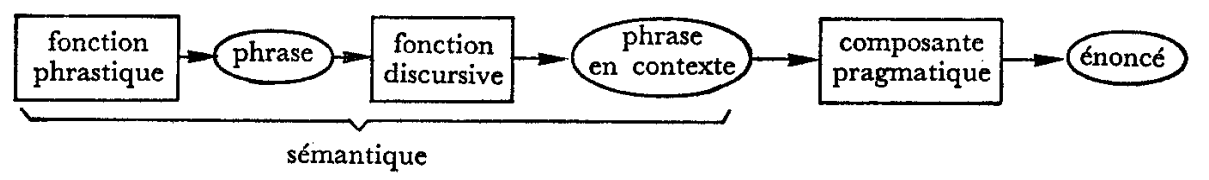

Figure 1 : Fac-similé du schéma de MARTIN (1983:15)

Cependant, au dernier chapitre de son ouvrage (dans l'édition de 1983), R. Martin va préciser et même modifier son schéma, en donnant aux deux précédentes fonctions un statut de «composante»; ce qui fait maintenant un système (toujours évolutif mais) à trois niveaux ou paliers :

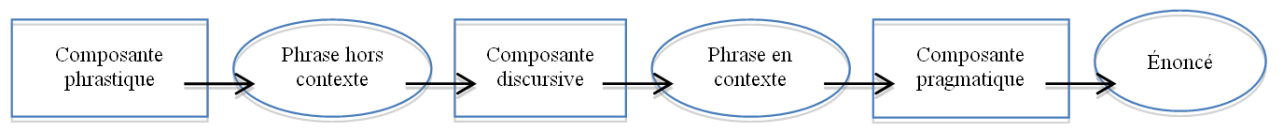

On peut toutefois penser que les deux schémas se complètent, plus qu'ils ne s'annulent l'un l'autre, dans la mesure où le «sémantique » du premier schéma peut fort bien réapparaître, pour continuer d'embrasser les deux anciennes «fonctions » devenues les deux premières « composantes » :

\footnotetext{
${ }^{19}$ Elle est aussi une interprétation et une adaptation d'O. DUCROT (1972; voir supra note17).

20 Aussi retrouve-t-on cette notion de «fonction», et le principe pluriel de diverses fonctions langagières, chez un autre grand auteur néo-pragois, M.A.K. Halliday, dont les travaux, dès les années 70, sont reconnus comme fondateurs de la linguistique discursive. Ceci dit, il ne faut pas oublier, comme le rappelle B. COMBETTES (1983), qu'en la matière K. Bühler est un prédécesseur de Jakobson. Quant à nous, il importe de rappeler que pour les trois auteurs fonctionnalistes cités, la fonction référentielle (informative) prime.
} 


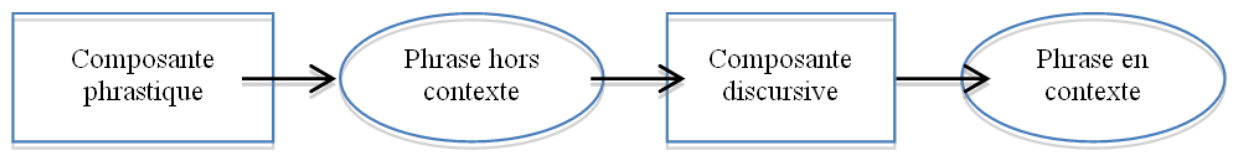

Il y a toutefois dans cette évolution du schéma une autre nouveauté : en parlant de phrase «hors» vs «en contexte», puis en attachant d'une part «hors contexte» à la «composante discursive » (du «sémantique»), d'autre part «en contexte » à la «composante pragmatique », R. Martin nous permet de préciser ce qu'il entend par contexte, et, par contrecoup, par discours : son hors contexte est ce nous appelons maintenant le cotexte; le discours (de sa composante discursive) est ce qu'aujourd'hui nous, linguistes du texte, appellerions le texte - suivant la dichotomie texte-discours que fixeront les travaux J.-M. Adam dans les années $80^{21}$.

D'ailleurs, dans le même chapitre le mot texte apparaît bien, lorsque R. MARTIN définit la «composante discursive » comme la phase «où la phrase s'insère dans la cohésion du texte »; même si l'assimilation texte-discours persiste, puisque quelques lignes plus bas il reformule sa proposition précédente ainsi : «située à mi-chemin de la composante phrastique et de la composante pragmatique, la composante discursive prend en charge la phrase hors contexte et l'intègre dans le discours » (op. cit. éd. 1983 : 204, éd. 1992 : 227).

Certes l'assimilation texte-discours peut nous sembler aujourd'hui une confusion ; on préfère en trouver la raison dans l'évolution ou l'histoire des idées et des théories linguistiques: R. Martin s'occupe plus de sémantique et encore de logique que de textualité; dans ce cadre logico-sémantique qui cherche le «naturel », la distinction texte-discours ou contexte-contexte n'apparaissent pas prioritaires, à l'inverse de celles que R. Martin pose entre "phrase » et «énoncé », « hors contexte» et « en contexte».

Pour autant, lorsqu'il s'agit de «cohésion », les choses se précisent : à cette notion de cohésion est bien attachée celle de texte, tel qu'il apparaît dans le raisonnement de R. MARTIN (op. cit. éd. 1983 : 204 et supra) ; à cette occasion, la notion de contexte est précisée ou redéfinie notamment par le passage ou l'évolution de ce que j'introduis infra sous [C1] et [C2] :

«Les phrases ne sont pas seulement plus ou moins conformes à la grammaire de la langue et aux exigences de la construction sémantique. Elles s'adaptent aussi plus ou moins harmonieusement au contexte [C1] où on les fait apparaître. Il importe ainsi de compléter [je souligne] la notion d'acceptabilité (grammaticalité et sémanticité) par celle de cohésion: la cohésion détermine l'appropriation d'une phrase bien formée à un contexte [C1bis]. Un texte [je souligne de nouveau] répond aux exigences de cohésion [id.] si toutes les phrases qu'il comporte y sont acceptées comme des suites possibles du contexte [C2] précédent. » (op. cit. éd. $1983: 204$, éd. $1992: 227)^{22}$.

${ }^{21}$ J.-M. ADAM (2010) étudie l'apport de R. Martin à la sémantique textuelle française des années 80.

${ }^{22}$ Remarque à propos de «suite possible »: R. MARTIN veut non seulement fonder une logique du langage naturel, il veut également une sémantique «relationnelle » et par là relative : «il ne s'agit 
Dans la foulée, c'est une autre distinction qu'opère R. Martin, entre cohésion et cohérence ; on peut suivre là encore l'évolution pas à $\operatorname{pas}^{23}$ :

«Ainsi la phrase, parfaitement acceptable :

Sophie, Pierre voudrait l'épouser

ne saurait servir de réponse à :

Qui Pierre voudrait-il épouser?

La cohésion du dialogue s'y oppose.

Au demeurant, la cohésion textuelle $e^{24}$, propre à la composante discursive, se complète, dans la composante pragmatique, par les exigences de la cohérence ${ }^{25}$. Celle-ci fait intervenir le contexte dans un sens plus large, c'est-à-dire la situation extra-linguistique et les connaissances d'univers. »

(op. cit. éd. 1983 : 204-205, éd. 1992 : 227-228 ; les italiques sont de mon fait pour souligner l'évolution dont je parlais ${ }^{26}$ )

Pour clore cette partie, je dirai que si R. Martin instaure un palier autonome entre phrase et discours, le texte, il continue néanmoins de considérer ce dernier comme relevant du domaine sémantique, et non pragmatique ; R. Martin fonde de la sorte une sémantique textuelle, comme le confirme d'ailleurs la citation qui conclut sa démonstration, et qui conclura la nôtre, dont nous retiendrons encore deux choses :

I. C'est là (op. cit. éd. 1983 : 206, éd. 1992 : 228) qu'apparaît son hommage explicite à l'École de Prague ${ }^{27}$ :

«Lieu de la "dynamique communicative" (École de Prague), de la "fonction textuelle" (M.A.K. Halliday), la composante discursive calcule donc l'adéquation de la phrase à son contexte.

En ce sens elle appartient à la sémantique. »

II. C'est encore à ce moment-là qu'il oriente son étude vers la notion de thème. Mais il le fait sous une forme à la fois inattendue pour nous aujourd'hui, et parfaitement cohérente avec sa démarche et son enracinement logico-sémantique :

«La cohésion textuelle se fonde sur des critères comme ceux d'isotopie, d'anaphore, de communauté présupositionnelle, dont la fonction s'exerce à l'intérieur même du texte, indépendamment de toute variation situationnelle.» (ibid.; je souligne communauté présupositionnelle, $\mathrm{R}$. Martin à l'intérieur même du texte).

pas de dire quel est le sens d'une phrase quelconque $p$, mais quelles relations de vérité unissent $p$ à d'autres phrases concevables » (Introduction, éd. $1983: 12,1992: 14$ ).

${ }^{23}$ Voir également en annexe 1 son schéma conclusif.

${ }^{24}$ Note de l'auteur : « Voir en partic. Charolles 1978 ».

${ }^{25}$ Autre note de R. Martin : «Proposition comparable dans Slakta 1975, p. 31-32».

${ }^{26}$ Remarquer en outre dans cette citation l'emploi de l'expression «connaissances d'univers » au lieu des habituelles «connaissances encyclopédiques », et la saisie même, indéfinie, du complément dans « connaissances d'univers » : R. Martin récupère ainsi la notion fondamentale de sa théorie que nous avons présentée plus haut, celle d' « univers », qui dans le contexte de sa démonstration, et dans son système logico-sémantique, subsume celle des « univers (de croyance) », c'est-à-dire tels qu'ils sont, au pluriel, attachés à des énoncés effectifs ou des énonciations particulières. Ceci dit, le fait qu'au singulier ou au pluriel, croyance remplace encyclopédie, montre, comme nous l'avons déjà dit, le tour psychologique que prend la sémantique logique de cet auteur, à la recherche du «naturel ».

${ }^{27}$ La citation que je donne est immédiatement précédée dans le texte de R. Martin par le schéma mis en annexe 1 . 
Arrivé à ce point, où apparaît le thème, je suspends mon analyse, gardant pour une prochaine étude (2012) l'articulation entre communauté présuppositionnelle et thème... Je vais donc conclure.

\section{Conclusion}

C'est finalement dans cette attirance-défiance du sémanticien logicien par et pour la pragmatique quasi naissante, avant même qu'elle ne devienne dominante, que se trouve me semble-t-il l'originalité de l'ouvrage de R. Martin quelle que soit son édition, tant il est vrai qu'il varie peu sur ce point ${ }^{28}$.

Attirance qui le mène non seulement de la phrase au texte, mais, au-delà encore, du texte à l'énoncé et du texte au contexte; défiance, cependant, qui le fait revenir à une forme de contexte "décontextualisé » : soit à une sémantique élargie certes au cotexte, mais dans la mesure où ce cotexte, dit «contexte antécédent », est fixe, stable, non soumis aux variables situationnelles.

Pour autant, il n'y a pas dans cette démarche, selon moi, de recul ou de revers ; mais bien une avancée épistémologique: sans le proclamer, R. Martin participe sinon à la fondation du moins à l'essor d'une linguistique textuelle, qu'il reviendra sans conteste à J.-M. Adam (accompagné parfois d'A. Petitjean), à M. Charolles et B. Combettes (parfois ensemble), de faire fructifier longtemps encore, après lui.

Hors texte, il y a une autre avancée chez R. Martin ; elle concerne ce que j' ai rassemblé, par commodité, sous le terme de psychologie : on reconnait aujourd'hui la parenté qui existe entre ses univers de croyances et les espaces mentaux de G. Fauconnier, même si seuls ces derniers ont connu le développement, le succès que l'on sait en sémantique cognitive ; or cette autre avancée ne nous éloigne pas tant de la précédente, et du propos de notre étude présente : on a entrevu en effet le lien entre présupposition (ou communauté de sens) et thème qu'instaure R. Martin en dernier lieu ; c'est une affaire dont je compte m'occuper, je l'ai dit, par la suite (2012).

Pour l'heure je voudrais finir sur un autre type d'héritage pragois que je trouve chez notre auteur. Dans son parcours, disons, prudent qui le conduit de la sémantique à la pragmatique, et son souci de ménager ou préserver la première, on peut voir une manière de prolonger ou de perpétuer la tradition immanentiste de la linguistique saussurienne, la linguistique comme discipline naissante :

«Le domaine de la sémantique est si vaste qu'on risque de s'y égarer et, pire, de le déserter ${ }^{29}$, sans trop s'en rendre compte, pour le terrain vague de la pragmatique tel qu' on peut l'entrevoir à la fin de l'ouvrage. Quelque discret qu'il soit, le fil d'Ariane des relations de vérité maintiendra dans l'immanence des phénomènes linguistiques. »

(Ce sont les derniers mots de l'introduction à $1983: 17$, comme à $1992: 19)$.

\footnotetext{
${ }^{28}$ Je remercie J.-M. Adam qui m'a suggéré lors de la journée Conscila 2010 de comparer les deux éditions, en guise de «vérification » de mes analyses qui portaient tout d'abord essentiellement sur la logique du sens version 1983.

${ }^{29}$ Prémonition, si l'on considère le développement de la pragmatique et la position aujourd'hui dominante des analyses du discours face à la sémantique du texte.
} 
Or, c'est le souci me semble-t-il que l'on trouve aussi dans l'École de Prague naissante, notamment chez V. Mathesius, son instigateur, tel que S. Raynaud (2008 : 51) le montre, attaché aux régularités du système :

« The sentence is not entirely the product of a transitory moment, is not entirely determined by the individual situation, and, consequently, does not entirely belong to the sphere of speech, but depends in its general form on the grammatical system of the language in which it is uttered. » (MATHESIUS, 1936, in : VACHEK, $1966: 317$ ).

\section{BIBLIOGRAPHIE}

ACHARD-BAYLE Guy (2001), Grammaire des métamorphoses. Référence, Identité, Changement, Fiction, Bruxelles, De Boeck-Duculot.

ACHARD-BAYLE Guy (2009), From the Prague Linguistic Circle to the French Text Grammar, conférence du 25/11/2009 à l'Université de Bohême du Sud et la Faculté de philosophie pour le colloque University of South Bohemia in a World of Science Without Borders, et la commémoration du $20^{\text {ème }}$ anniversaire de la Révolution de velours. Actes publiés en 2011 par l'UBS, CD-Rom.

ACHARD-BAYLE Guy (2010), Du Cercle de Prague à une linguistique du texte «à la française », Annonce des journées sur la Perspective fonctionnelle de la phrase Metz-Nancy 2012 \& Plaidoyer pour une géo-politique des sciences, in : G. ACHARD-BAYLE \& S. RAYNAUD (a cura di), Actes de la Journée à l'Université catholique de Milan Le Cercle linguistique de Prague : Centre et périphérie, paru dans : L'Analisi linguistica e letteraria, XVIII, p. 431-436.

ACHARD-BAYLE Guy (éd., à par. 2012), Introduction aux Actes de la Journée Conscila (Confrontation en sciences du langage) du 28 mai 2010 à l'ENS Ulm Paris, Linguistique textuelle : états de lieux, Actes à par. in : revue Verbum, Nancy, PU.

ADAM Jean-Michel (1977), Ordre du texte, ordre du discours, Pratiques 13, Textes-Linguistique, p. 103-111.

ADAM Jean-Michel (2005, autres éd. en 2008 et 2011), La linguistique textuelle. Introduction à l'analyse textuelle des discours, Paris, A. Colin.

ADAM Jean-Michel (2006), Texte, contexte et discours en questions, in: Entretiens avec Guy Achard-Bayle (éd.), Pratiques 129-130, TextesContextes, p. 20-49.

ADAM Jean-Michel (2010), Conférence du 28 mai à l'ENS Ulm Paris, Perspective fonctionnelle de la phrase et progressions thématiques. Des débuts de la linguistique textuelle en France à aujourd'hui. Rôles de Denis Slakta et Bernard Combettes (1975-2010), à par. in : Actes de la journée Conscila, G. Achard-Bayle (éd.). Linguistique textuelle: états de lieux, Nancy, PU, revue Verbum.

ARNAULD Antoine \& LANCELOT Claude (1660), Grammaire générale et raisonnée, sur le site de la Bibliothèque nationale de France, Gallica: <http://gallica.bnf.fr/ark:/12148/bpt6k843201>. 
Charolles Michel (1976), Grammaire de texte-théorie du discours-narrativité, Pratiques 11-12, Récit (i), p. 133-154.

Charolles Michel (1978), Introduction aux problèmes de la cohérence des textes, Langue française 38, p. 7-41.

CHAROLles Michel (1997), L'encadrement du discours Univers, Champs, Domaines et Espaces, Publications de l'université Nancy 2, Laboratoire Landisco (Langage, discours, cognition), Cahiers de recherches linguistiques 11. Et en ligne sur le site du laboratoire LaTTICe : http://www.lattice.cnrs.fr/Michel-Charolles, 219.

CHOMsky Noam (1966), Cartesian Linguistics. A Chapter in the History of Rationalist Thought, traduction fr. 1969, Linguistique cartésienne. Un chapitre de l'histoire de la pensée rationaliste, Paris, Éd. du Seuil.

CombetTES Bernard (1976), recension de : Teun Van Dijk, Some Aspects of Text Grammars, in Notes de lecture, Pratiques 11-12, Récit (i), p. 197-203.

COMBetTes Bernard (1983), Pour une grammaire textuelle. La progression thématique, Bruxelles, De Boeck-Duculot \& Metz, revue Pratiques.

COMBETTES Bernard \& FRESSON Jacques (1975), Quelques éléments pour une linguistique textuelle, Pratiques 6, p. 25-55, en ligne : http://www.pratiquescresef.com/p006_co1.pdf.

COMBETTES Bernard (2010), conférence du 28 mai à l'ENS Ulm Paris, La «linguistique du texte » telle qu'elle a commencé à se développer en France dans les années 70, à par. in Actes de la journée Conscila, G. Achard-Bayle (éd.). Linguistique textuelle : états de lieux, Nancy, PU, revue Verbum.

Combettes Bernard (2011), Phrase et proposition. Histoire et évolution de deux notions grammaticales, Le Français aujourd'hui 173, p. 11-20.

DANEŠ František (ed. 1974), Papers on functional sentence perspective. Praha, Academia. (Voir notamment: Functional sentence perspective and the organization of the text, p. 106-128).

DUVAL Frédéric (éd. 2011), La logique du sens : autour des propositions de Robert Martin, Actes du Colloque R. Martin, Université de Metz mars 2011, Publications de l'université de Metz, Recherches linguistiques.

FAUCONNIER Gilles (1984), Espaces mentaux, Paris, Les Éditions de Minuit. (Trad. angl, Mental Spaces: Aspects of Meaning Construction in Natural Language, first published in 1985, MIT Press, reprint 1994, Cambridge, Cambridge University Press).

FERRET Stéphane (1996), Le bateau de Thésée. Le problème de l'identité personnelle à travers le temps, Paris, Les Éditions de Minuit.

FIRBAS Jan (1974), Some aspects of the Czechoslovak approach to problems of functional sentence perspective, in : F. DANEŠ (ed.), p. 11-37.

HoskOVEC Tomáš (2010), Linguistique textuelle et le programme de philologie englobante, à par. in : Actes de la journée Conscila, G. ACHARD-BAYLE (éd.). Linguistique textuelle : états de lieux, Nancy, PU, Verbum.

MARTIN Robert (1976), Inférence, antonymie et paraphrase, Publications du Centre d'Analyses Syntaxiques Université de Metz \& Paris, Klincksieck.

MARTIN Robert (1983, 2 ème éd. revue et augmentée 1992), Pour une logique du sens, Paris, PUF. 
MATHESIUS Vilém, 1936, Deset let Pražského linguistického kroužku, Slovo a slovesnost 2, p. 137-145. Trad. angl. in : J. VACHEK (ed., 1966), p. 137-151.

PEŠEK Ondřej (2010), La linguistique textuelle tchèque au seuil du XXI ${ }^{\mathrm{e}}$ siècle : tradition, inspirations, ouvertures, à par. in : Actes de la journée Conscila, G. ACHARD-BAYLE (éd.). Linguistique textuelle : états de lieux, Nancy, PU, Verbum.

RAYNAUd Savina (1990), Circolo Linguistico di Praga, Milan, Pubblicazioni dell'Università Cattolica di Milano.

RAYNAUD Savina (2008), The basic syntagmatic act is predication, Slovo a slovestnost, 1-2, 69, p. 49-66.

SlaKTA Denis (1975), L'ordre du texte, Études de linguistique appliquée 19, p. 30-42.

VACHEK Josef (ed. 1966), The Linguistic School of Prague: An Introduction to its Theory and Practice, Bloomington, Indiana UP, p. 137-151.

\section{Annexe 1}

Fac-similé de Martin (1983 : 206, 1992 : 228)

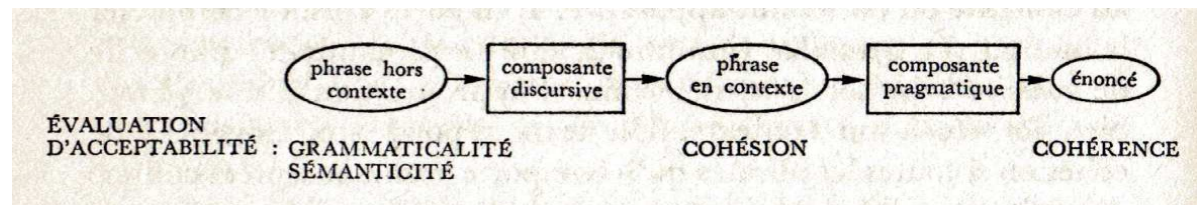

